- This paper investigates a new orthodontic occlusal index, the Index of Outcome, Complexity and Need (ICON). It suggests that this single index can replace the PAR index and the Index of Orthodontic Treatment Need (IOTN). The implications are:

- The single index (ICON) which is easier to learn and use than PAR and IOTN could measure orthodontic treatment need and treatment standards.

- The index could be easily incorporated into the orthodontic assessment of a patient in general dental practice.

- The inclusion of IOTN in the general dental services has been proposed. ICON would be quicker to learn, apply and more information could be gleaned than just treatment need.

\title{
A comparison of the Index of Complexity Outcome and Need (ICON) with the Peer Assessment Rating (PAR) and the Index of Orthodontic Treatment Need (IOTN)
}

\author{
N. A. Fox ${ }^{1}$ C. Daniels ${ }^{2}$ and T. Gilgrass ${ }^{3}$
}

\begin{abstract}
Aim To evaluate any relationship between ICON, IOTN and PAR. To establish whether or not ICON could replace these indices as a measure of orthodontic treatment complexity, outcome and need.

Method The study models of 55 consecutively treated cases were examined and PAR, IOTN and ICON recorded.

Results The study showed significant correlations between IOTN and ICON with respect to need and PAR and ICON with respect to outcome. Conclusion It appears that ICON does reflect UK opinion and the current study provides some evidence that ICON may effectively replace PAR and IOTN as a means of determining need and outcome.
\end{abstract}

The PAR Index and IOTN have now become widely used in the UK as orthodontic audit tools. They have provided valuable data to inform political and clinical debate on the quality of GDS orthodontics. The introduction of IOTN in the General Dental Services is currently being considered. In order to assess treatment inputs and outcomes using IOTN and PAR, two different measurement protocols must be learned and this duplication of effort is inefficient.

The PAR index has been accused of both undue leniency on poor finishes ${ }^{1}$ and undue harshness on treatments with limited aims. $^{2,3}$ Experience with IOTN has shown that the need for treatment does not necessarily equate to the complexity of the treatment. It is important to make an assessment of complexity for the following clinical reasons (Richmond et $a l^{4}$ ):

- To identify the most appropriate setting in which a patient should receive treatment.

- To allow meaningful assessment of treatment outcomes.

- To identify cases that are likely to take longer to treat.

- To inform the patient of the likely success.

\footnotetext{
${ }_{1 *}$ Consultant Orthodontist, Orthodontic Department, Middlesbrough General Hospital, ${ }^{2}$ Lecturer in Orthodontics, Department of Dental Health and Development, University Dental Hospital, NHS Trust, Heath Park, Cardiff. ${ }^{3}$ Consultant Orthodontist, Orthodontic Department, Newcastle Dental Hospital,

${ }^{*}$ Correspondence to: Nigel Fox, Orthodontic Department, Middlesbrough General Hospital, Ayresome Green Lane, Middlesbrough, Cleveland TS5 5AZ

Email:nigel.a.fox@btinternet.com
}

\section{Refereed paper}

Received 14.06.01; Accepted 19.04.02

๑ British Dental Journal 2002; 192: 225-230
Richmond et $a l^{4}$ found that the factors which may determine the professional perception of orthodontic treatment complexity include:

- Cost of treatment

- Number of appointments

- Length of appointments

- Age of patient when treatment commenced

- Initial PAR Score

However they discounted most of these treatment factors as litthe use in the formulation of a complexity index because they were too dependent on the choices and efficiency of the clinician. The PAR score was suggested to be an interim possibility in the development of a complexity index.

The ICON index ${ }^{5}$ has been developed recently and claims among other things, to evaluate orthodontic treatment complexity. ICON is based on the subjective judgements of 97 orthodontists from nine countries on 240 initial and 98 treated models. ${ }^{5}$ The index is described in full in the appendix. In brief, the index comprises five weighted measurements, and owes some of its structure to IOTN and PAR. The measured traits include:

- Dental aesthetics as measured by the aesthetic component of IOTN. ${ }^{6}$

- The presence of a crossbite

- Anterior vertical relationship ie deep bites and open bites as measured by PAR. ${ }^{7}$

- Upper arch crowding/spacing on a five point scale

- Buccal segment antero-posterior relationship as measured by PAR. $^{7}$

The sum of the weighted scores is interpreted using cut-off values and score ranges to indicate the treatment need, complexity and degree of improvement. The cut-off values are to give the index meaning and link the numerical ICON score to the original subjective description of the 97 orthodontists. It is claimed that using ICON to make these assessments is more efficient (than PAR and IOTN) because it requires only a single measurement protocol. ICON should prove to be a useful tool for research and audit, but if used alongside PAR and IOTN, could be an additional burden in a busy hospital or practice setting. 
PAR and IOTN have been validated against UK dental opinion $^{7,8}$ and in an increasingly global environment, perhaps a more internationally recognised standard should now be sought. ICON reflects the views of 97 orthodontists from eight European countries and the USA, and may provide an acceptable alternative to IOTN and PAR.

The aim of this paper is to investigate any link between PAR, IOTN and ICON with respect to orthodontic treatment need and outcome.

\section{METHOD}

A retrospective sample of 55 consecutively treated cases was identified from the orthodontic department of Middlesbrough General Hospital. Cases were treated by clinical assistants, specialist registrars or one of three consultant orthodontists. Discontinued cases were included in the sample.

Pre-treatment and post-treatment study models were scored using IOTN, PAR and ICON by a calibrated examiner from another hospital (TG). The examiner did not know which operator was responsible for treating the case when the models were scored. Pre- and post-treatment models were scored at random

\section{Data analysis}

Descriptive statistics were calculated to locate the central tendency and spread. Intra-examiner reliability was examined by repeating the examination of a random subset of 15 pre-treatment and post-treatment cases. Reliability was expressed using the weighted Kappa statistic for ordinal scores (IOTNDHC, IOTNAC, ICON complexity). The reliability of ICON scores and PAR were expressed using Root Mean Square to assess random error and a Students $t$-test for systematic bias. The relationship between ICON and the other indices was examined using nonparametric (Spearman) correlation.

The correlations were calculated for the pre-treatment and post-treatment data separately.

Outcome assessment has been traditionally made by assessing either the percentage change or absolute change in occlusal index scores. Assessment of occlusal improvement with ICON is made by subtracting $4 \mathrm{x}$ post-treatment score from the pre-treatment score, ${ }^{5}$ with the range of possible results nominally given five categories of improvement. These five categories can be thought of as similar in purpose to the three PAR improvement grades as determined from the PAR nomogram.

Bivariate correlations were calculated for PAR reduction versus ICON improvement and per cent PAR reduction versus ICON improvement.

\section{RESULTS}

The reliability assessment of the indices is given in Tables 1a and $1 \mathrm{~b}$ and descriptive statistics are given in Tables $2 \mathrm{a}$ and $2 \mathrm{~b}$. When used as an ordinal scale to express treatment complexity, ICON has marginally lower but acceptable reliability as the use of IOTN to assign treatment need categories. When used as a quasi-continuous variable (similar to PAR) it has a similar level of precision.

\begin{tabular}{lccc}
\multicolumn{4}{l}{ Table 1a Kappa values for Intra-examiner reliability } \\
\hline Complexity & AC & DHC & ICON \\
\hline Kappa & & & \\
Lower 95\% confidence limit & 0.88 & 0.82 & 0.78 \\
Upper 95\% confidence limit & 0.92 & 0.71 & 0.68 \\
\end{tabular}

\begin{tabular}{lcc}
\multicolumn{3}{l}{ Table $1 \mathbf{b}$ Differences in values for Intra-examiner reliability } \\
\hline & PAR & ICON \\
\hline Mean difference & 1.6 & 1.83 \\
P(t-test) & 0.086 & 0.588 \\
rms (random) error & 2.33 & 7.9
\end{tabular}

$\begin{aligned} & \text { Table 2a } \\
& (\mathrm{n}=55)\end{aligned}$
\begin{tabular}{lcc} 
Index & Pre-treatment median & Post-treatment median \\
\hline IOTN (AC) & 8 & 2 \\
IOTN (DHC) & 4 & 2
\end{tabular}

\begin{tabular}{|c|c|c|}
\hline Index & Pre-treatment mean (S.D.) & Post-treatment mean (S.D.) \\
\hline PAR & $38.2(10.6)$ & $5.4(5.9)$ \\
\hline ICON & $72.9(13.0)$ & $18.4(7.9)$ \\
\hline
\end{tabular}

The RMS value appears to be three times higher for ICON compared with PAR. This is because the minimum score increment for ICON is three times higher than PAR.

Table 3 shows the correlations between ICON, IOTN and PAR. The IOTN scores suggest that the sample showed a definite need for treatment on the grounds of dental health and aesthetics and that the standard of treatment was good. Figure 1 shows the plot of DHC versus ICON. For any given IOTN grade there are a range of possible ICON scores. As expected DHC and ICON scores are higher for pre-treatment cases. Both IOTN (DHC) and ICON classified two pre-treatment cases into the no treatment category, ie $98 \%$ of the sample justifies treatment on the grounds of dental health as defined by the UK usage of the DHC or according to the international standard set by ICON. Most of the post-treatment models score an acceptable end result as defined by ICON $^{5}$ ie below 31. The poor correlation of ICON and DHC scores is related to the limited ordinal scale of DHC with most pre-treatment cases falling in grades 4-5 and post treatment 2-4. Many post treatment IOTN (DHC) scores were grade 4 and 3. This suggests a poor treatment result not reflected by IOTN (AC), PAR or ICON. This is because the study model protocol was applied and consequently any case with any crossbite anywhere in the mouth would have to be awarded grade 4 regardless of the size of the displacement. Most post-treatment grade 3 IOTN (DHC) scores were due to second molars not being routinely banded. This resulted in a small contact point displacement between first and second molars, which meant grade 3 had to be awarded. PAR or ICON does not pick this up.

Figure 2 shows the plot of IOTN (AC) versus ICON. For any given IOTN (AC) grade there are a range of possible ICON scores. According to the UK usage of IOTN (AC), 30\% of this sample would not be justified for definite treatment need on the grounds of aesthetics alone (ie an AC score of 1 to 7).

Figure 3 shows a plot of PAR score against ICON for pre- and post-treatment study casts. It can be seen that there is a good level of agreement between PAR and ICON.

Figure 4 compares the outcome assessment of per cent PAR reduction with ICON improvement. Overall the relationship has a significant (Pearson) correlation of 0.74. Approximately 55\% of the sample would be classified as greatly improved using ICON. The mean per cent PAR reduction is 85.0 and the mean PAR reduction is 32.8 , suggesting the sample was very well treated compared with previous UK samples and in terms of international standards set by ICON.

Table 3 Spearman rank order correlation coefficients for AC, DHC and ICON scores for pre and post treatment cases

\begin{tabular}{lcc}
\hline $\begin{array}{l}\text { Spearman correlations } \\
\text { between Indices }\end{array}$ & Pre-treatment models & Post-treatment models \\
\hline & & \\
ICON versus IOTN DHC & 0.17 & 0.46 \\
ICON versus IOTN AC & 0.64 & 0.86 \\
ICON versus PAR & 0.51 & 0.36
\end{tabular}




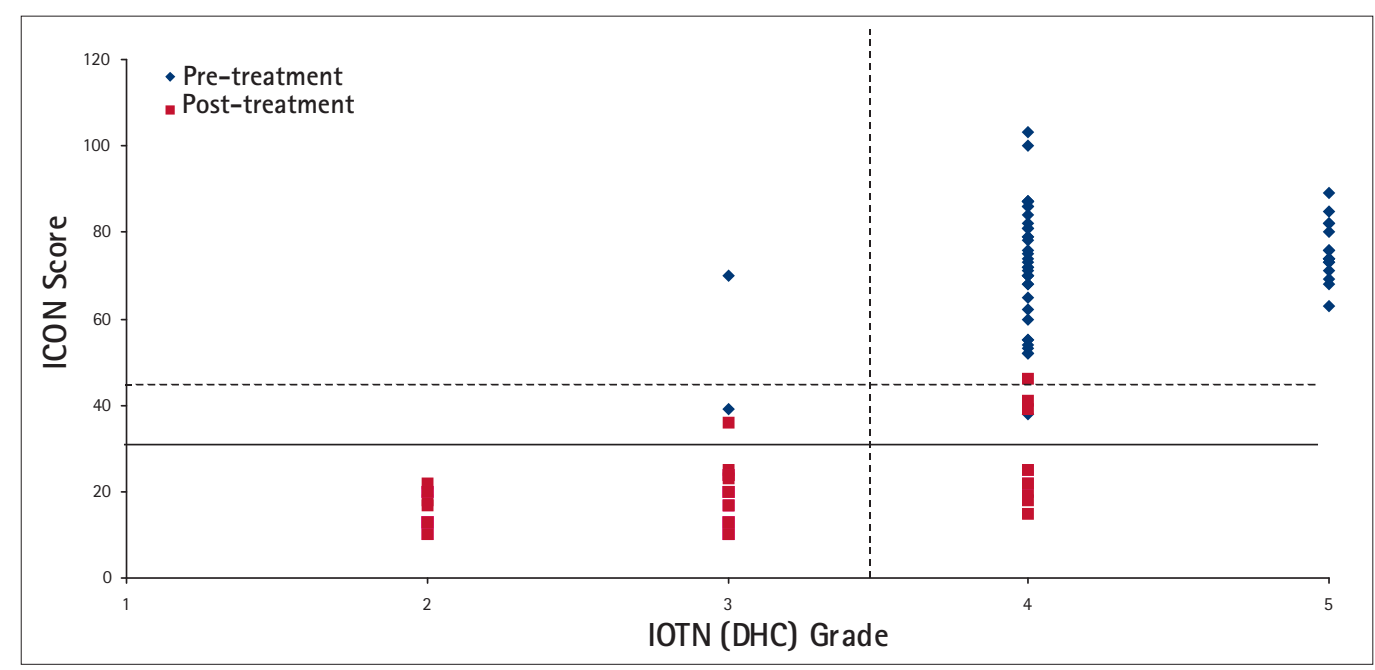

Figure 1 Scatter plot of ITON (DHC) versus ICON for pre-treatment and post-treatment casts.

A dashed line denotes treatment thresholds. For IOTN the treatment category is for grades 4 and 5 , and for ICON is any score greater than 43. The dotted line signifies the ICON value (31) below which a treatment can be considered acceptable. The possible score for DHC ranges $1-5$ and $7-128$ for ICON.

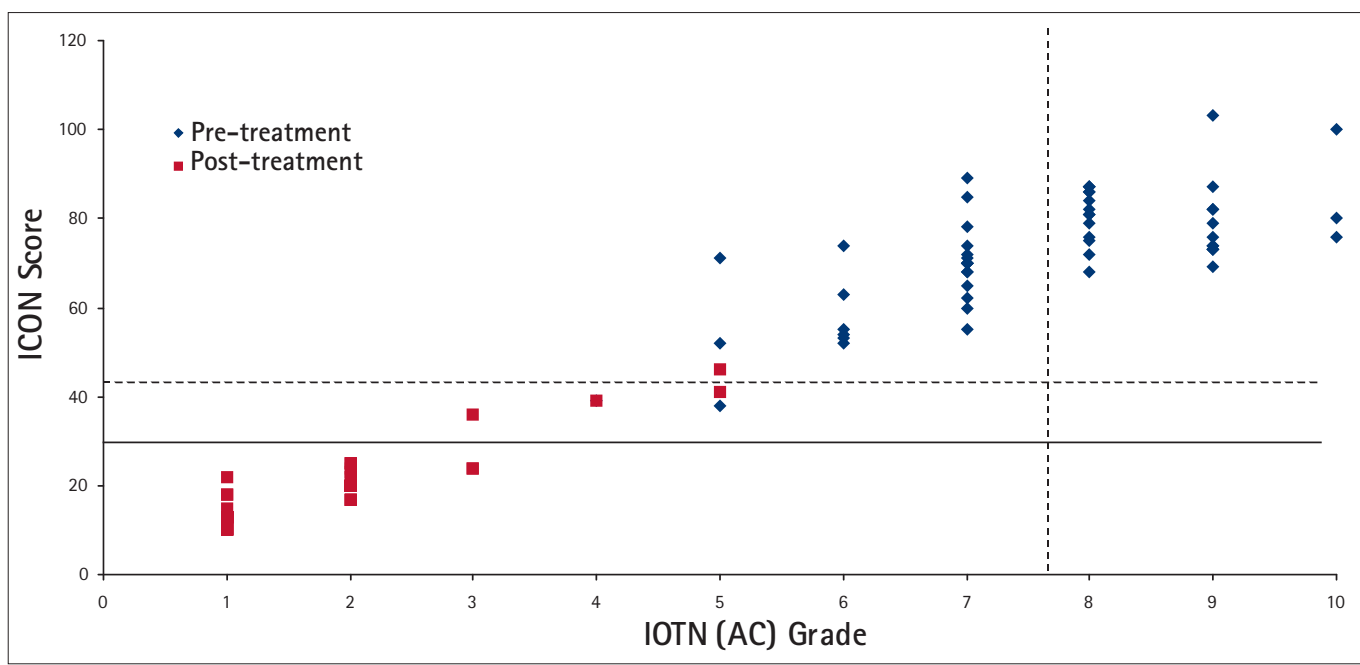

Figure 2 Scatter plot of IOTN (AC) versus ICON for pre-treatment and post-treatment casts.

A dashed line denotes treatment thresholds. For IOTN (AC) the treatment category is for grades $8-10$, and for ICON is any score greater than 43 . The solid line signifies the ICON value (31) below which a treatment can be considered acceptable. The possible score for $A C$ ranges $1-10$ and $7-128$ for ICON.

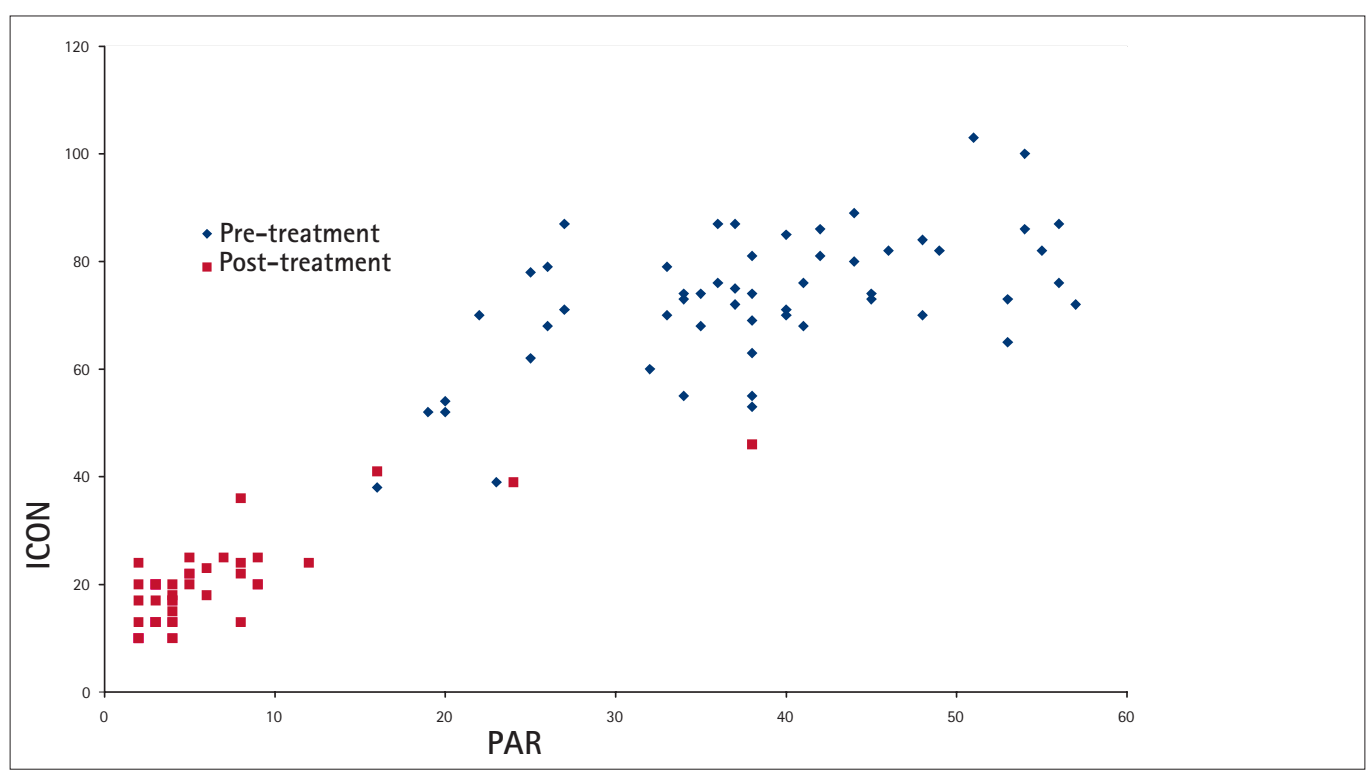

Figure 3 Scatter plot of PAR versus ICON for pre-treatment and posttreatment models.

\section{DISCUSSION}

The sample used for this comparison was relatively small but reasonably diverse in as much as a fairly broad range of treatment starts were obtained and treatment was delivered by a variety of expert levels within the hospital service. The overall level of malocclusion is however more severe than previous estimates from the GDS, which typically have had mean initial PAR scores of $29 .{ }^{9}$ The sample, however, was not designed to generalise treatment outcomes in the GDS or hospital sector, but to compare the performance of the new indices.
Examination of Table 3 clearly shows that the degree of correlation between ICON and the other indices is influenced by the type of models in the sample. In this hospital sample, pre-treatment cases were moderately severe and most treatments resulted in a very minor malocclusion. For this reason a higher correlation is obtained when comparing index scores based on the pre-treatment and post-treatment dental casts. A lower level of correlation is reported for the assessment of treatment outcomes, probably because the treatment results were consistently good. (If there is little variation in a sample, poor correlations result). 


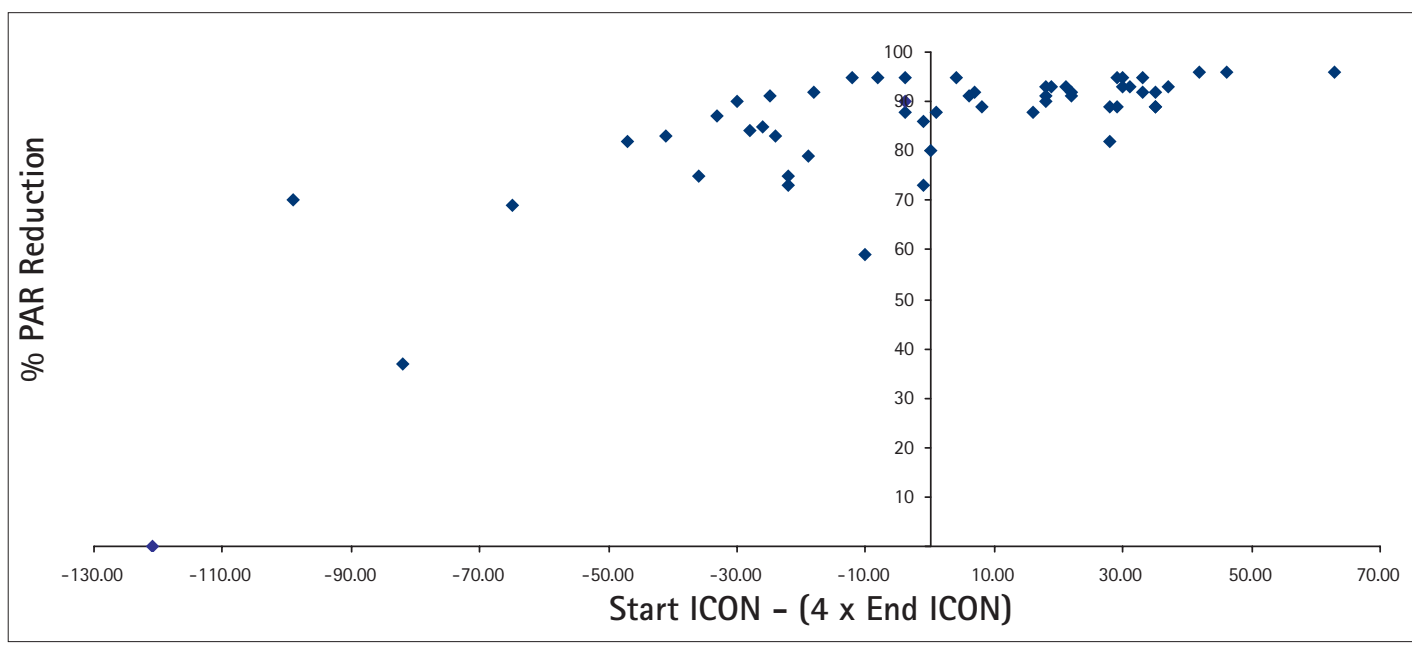

The general performance of the ICON index seems to be largely in agreement with IOTN for assessing treatment need. Although ICON identified a higher proportion of the sample in need of treatment compared with IOTN (AC), on which it is heavily based, it did not over score on the proportion of cases identified for treatment on the grounds of dental health. This suggests that ICON could probably substitute IOTN (DHC) and produce largely similar results. An ICON score of greater than 43 reflects a definite need for treatment. ${ }^{5}$

The general performance of the ICON index seems to be equivalent to the PAR for assessing treatment results. Both indices identified similar proportions of the most improved cases, but ICON overall is a more stringent standard to attain a 'greatly improved' categorisation. An ICON score of 31 or less defines a clinically acceptable result. ${ }^{5}$ Like all occlusal indices, ICON will not be appropriate in assessing need and outcome in a small number of cases. This is certainly true of PAR. ${ }^{1,2,3}$ Its use does, however, seem appropriate when examining a representative sample of cases from a single practitioner or hospital department.

\section{CONCLUSIONS}

1. ICON appears able to substitute IOTN (AC) and IOTN (DHC) as a means of measuring orthodontic treatment need. A value of greater than 43 for ICON defines a definite need for treatment.

2. There is a close relationship between PAR score and ICON. Its use in measuring treatment outcome appears to be as appropriate as using PAR.

3. ICON appears to require a more stringent standard than PAR to attain a 'greatly improved' categorisation. An ICON score of less than 31 defines a clinically acceptable result.

The authors would like to thank Alison Downing and Lynn May, Consultant Orthodontists at Middlesbrough General Hospital for their permission to examine records of patients under their care, the dental nurses for their help with data collection and Mrs M. Revelle for her help with the manuscript

1.Hinman C, The Dental Practice Board -the current status. Br J Orth 1996; 22: 287-290

2. Kerr J, Buchanan I B. The use of the PAR in assessing the effectiveness of removable orthodontic appliances. Br J Orth 1993: 20:351-357.

3. Fox N A. The first 100 cases: A personal audit of orthodontic treatment assessed by the PAR (Peer Assessment Rating) Index. Br Dent J 1993; 174: 290-297.

4. Richmond S, Daniels C P, Fox N A, Wright J. The professional perception of orthodontic treatment complexity. Br Dent J 1997: 183:365-370.

5. Daniels C P, Richmond S. The development of the Index of Complexity Outcome and Need (ICON). J Orthod 2000; 27: 149-162

6. Shaw W C, Richmond S, O'Brien K D, Brook P. Stephens C. Quality control in orthodontics. Indices of treatment need and outcome. Br DentJ 1991; 170: 107-112.

7. Richmond S, Shaw W C, O'Brien K D, et al. The development of the PAR Index (Peer Assessment Rating) reliability and validity. Eur J Orth 1992; 14: 125-139.

8. Richmond S, Shaw W C, O'Brien K D, et al. The relationship between IOTN and the consensus opinion of a panel of 74 dentists. Br Dent J 1995; 178: 370-374.

9. Fox N A, Richmond S, Daniels C, Wright J. Factors affecting the outcome of orthodontic treatment in the General Dental Services. Br J Orth 1997; 24: 217-21.
APPENDIX - THE INDEX OF COMPLEXITY, OUTCOME AND NEED Practical use of the index to assess treatment need

To use the index to assess treatment need the pre-treatment study models are examined and occlusal traits are scored according to the protocol below. The five occlusal trait scores are then multiplied by their respective weightings and summed (Table 4). If the summary score is greater than 43 , treatment is indicated.

Table 4 ICON index variables, weightings and cut-off values for treatment need and outcome decisions

Occlusal Trait ICON index weighting

IOTN Aesthetic 7

Component

Left + right buccal 3

Antero-posterior

Upper arch Crowding

Overbite

Crossbite

Treatment need cut-off

Treatment outcome cut-off

3

5

5

Practical use of the index to assess treatment outcome acceptability

To assess treatment outcome, apply the index scoring method to the post-treatment models only. If the summary score is less than 31 the outcome is acceptable.

Practical use of the index to assess treatment complexity

To assess treatment complexity, a five point scale is used via the cut points for the 20 percentile intervals, using the ranges given in Table 5 from the pre-treatment models.

Table 5 ICON index complexity cut-off values

\begin{tabular}{ll}
\hline Complexity grade & Score range \\
\hline Easy & less than 29 \\
Mild & 29 to 50 \\
Moderate & 51 to 63 \\
Difficult & 64 to 77 \\
Very difficult & greater than 77
\end{tabular}

Practical use of the index to assess the degree of improvement To assess the degree of improvement multiply the post-treatment score by 4 , and subtract the result from the pre-treatment score. Use the ranges in Table 6 to assign a grade.

When the index is used to assess treatment outcomes, it is assumed that an appropriate level of co-operation was obtained from the patient. The index may require confirmation of the presence of teeth using radiography. Except for the aesthetic assessment, occlusal traits are not scored to deciduous teeth unless they are to be retained in the permanent dentition to obviate the need for a prosthetic replacement, for example when the permanent tooth is absent.

The index contains five components all of which must be scored. 


\begin{tabular}{l}
$\begin{array}{l}\text { Table } 6 \text { Pre-treatment }-4 \text { (Post-treatment) ICON index score ranges, for } \\
\text { ratings of treatment improvement }\end{array}$ \\
\hline \begin{tabular}{ll} 
Improvement grade & Score range \\
\hline greatly improved & $>-1$ \\
substantially improved & -25 to -1 \\
moderately improved & -53 to -26 \\
minimally improved & -85 to -54 \\
not improved or worse & $<-85$
\end{tabular}
\end{tabular}

\section{Dental aesthetics}

The dental aesthetic component of the IOTN ${ }^{6}$ is used. The dentition is compared with the illustrated scale and a global attractiveness match is obtained without attempting to closely match the malocclusion to a particular picture on the scale (Fig. 5). The scale works best in the permanent dentition.

The scale is graded from 1 for the most attractive to 10 for the least attractive dental arrangement. Once this score is obtained it is multiplied by the weighting of 7 .

\section{Crossbite}

A normal transverse relationship in the buccal segments is observed when the palatal cusps of the upper molar and premolar teeth occlude preferably into the occlusal fossa of the opposing tooth or at least between the lingual and buccal cusp tips of the opposing tooth. Crossbite is deemed to be present if a transverse reaction of cusp to cusp or worse exists in the buccal segment.

This includes buccal and lingual crossbites consisting of one or more teeth with or without mandibular displacement.

In the anterior segment a tooth in crossbite is defined as an upper incisor or canine in edge to edge or lingual occlusion.

Where a crossbite is present in the posterior or anterior segments or both, the raw score of 1 is given which is multiplied by the weighting of 5 .

Where there is no crossbite the score for this trait is zero.

\section{Anterior vertical relationship}

This trait includes both open bite (excluding developmental conditions) and deep bite. If both traits are present only the highest scoring raw score is counted. Positive overbite is measured at the deepest part of the overbite on incisor teeth. Scoring protocol is given in Table 4.

Open bite may be measured with an ordinary millimetre rule to the mid-incisal edge of the most deviant upper tooth. Multiply the raw score obtained by 4 .

Retained deciduous teeth (ie without a permanent successor) and erupted supernumerary teeth should be scored as space unless they are to be retained to obviate the need for prosthesis. In transitional stages average canine and premolar widths can be used to estimate the potential crowding. Suggested averages are $7 \mathrm{~mm}$ for premolar and lower canine and $8 \mathrm{~mm}$ for upper canine. The presence of erupted antimeric teeth allows more accurate estimation for this purpose. Spacing due to teeth lost to trauma and exodontia is also counted.

Post-treatment spaces created to allow prosthetic replacements should match the antimeric tooth width. Discrepancy between such spaces and the anitmeric tooth can be counted as excess spacing or crowding, whichever is appropriate. The use of the index to assess spacing in relation to retained deciduous teeth demands that the fate of the deciduous teeth is known before the index can be applied.

Once the raw score has been obtained it is multiplied by the weighting 5 .

\section{Upper arch crowding/spacing}

This variable attempts to quantify the tooth to tissue discrepancy present in the upper arch or the presence of impacted teeth in both arches.

The sum of the mesio-distal crown diameters is compared with the available arch circumference, mesial to the last standing tooth on either side. This may require the use of a millimetre rule for accuracy, but with practice can be estimated by eye with reasonable accuracy.

No estimation is made to account for the curve of Spee or the degree of incisor inclination. Once the crowding/spacing discrepancy has been worked out in millimetres it is reduced on to the ordinal scale using the categories shown in Table 7.

Note that an impacted tooth in either the upper or lower arch immediately scores the maximum for crowding. A tooth must be unerupted to be defined as impacted.

An unerupted tooth is defined as impacted under the following conditions:

1. If it is ectopically placed or impacted against an adjacent tooth (excluding third molars but including supernumerary teeth).

2. When less than $4 \mathrm{~mm}$ of space is available between the contact points of the adjacent permanent teeth.

\section{Buccal segment antero-posterior relationship}

The scoring zone includes the canine premolar and molar teeth. The antero-posterior cuspal relationship is scored according to the protocol given in Table 7 for each side in turn. The raw scores for both sides are added together and then multiplied by the weighting 3 .

\section{Deviation of the final score}

Once all of the raw scores have been obtained and multiplied by their respective weights, they are added together to yield a single weighted summary score for a particular cast.

Table 7 Protocol for occlusal trait scoring

\begin{tabular}{|c|c|c|c|c|c|c|c|}
\hline & SCORE & 0 & 1 & 2 & 3 & 4 & 5 \\
\hline Aesthetic & $1-10$ as judged using SCAN & & & & & & \\
\hline Upper arch crowding & $\begin{array}{l}\text { Score only the highest trait } \\
\text { either spacing or crowding }\end{array}$ & $<2 \mathrm{~mm}$ & $2.1 \mathrm{~mm}-5 \mathrm{~mm}$ & $5.1-9 \mathrm{~mm}$ & $9.1-13 \mathrm{~mm}$ & $13.1-17 \mathrm{~mm}$ & $\begin{array}{l}>17 \mathrm{~mm} \text { or impacted } \\
\text { teeth }\end{array}$ \\
\hline Upper spacing & & Up to $2 \mathrm{~mm}$ & $2.1 \mathrm{~mm}-5 \mathrm{~mm}$ & $5.1-9 \mathrm{~mm}$ & $>9 \mathrm{~mm}$ & & \\
\hline Crossbite & $\begin{array}{l}\text { Transverse relationship of } \\
\text { cusp to cusp or worse }\end{array}$ & No crossbite & Crossbite present & & & & \\
\hline Incisor open bite & $\begin{array}{l}\text { Score only the highest trait } \\
\text { either open bite or overbite }\end{array}$ & Complete bite & $<1 \mathrm{~mm}$ & $1.1 \mathrm{~mm}-2 \mathrm{~mm}$ & $2.1-4 \mathrm{~mm}$ & $>4 \mathrm{~mm}$ & \\
\hline Incisor overbite & Lower incisor coverage & Up to $1 / 3$ tooth & $1 / 3-2 / 3$ coverage & $\begin{array}{l}2 / 3 \text { up to full } \\
\text { covered }\end{array}$ & Fully covered & & \\
\hline $\begin{array}{l}\text { Buccal segment } \\
\text { antero-posterior }\end{array}$ & Left and right added together & $\begin{array}{l}\text { Cusp to embrasure } \\
\text { relationship only. } \\
\text { Class I, II or III }\end{array}$ & $\begin{array}{l}\text { Any cusp relation up } \\
\text { to but not including } \\
\text { cusp to cusp }\end{array}$ & $\begin{array}{l}\text { Cusp to cusp } \\
\text { relationship }\end{array}$ & & & \\
\hline
\end{tabular}




\section{RESEARCH}

\section{Figure 5 The aesthetic component of IOTN used as the first component of ICON}
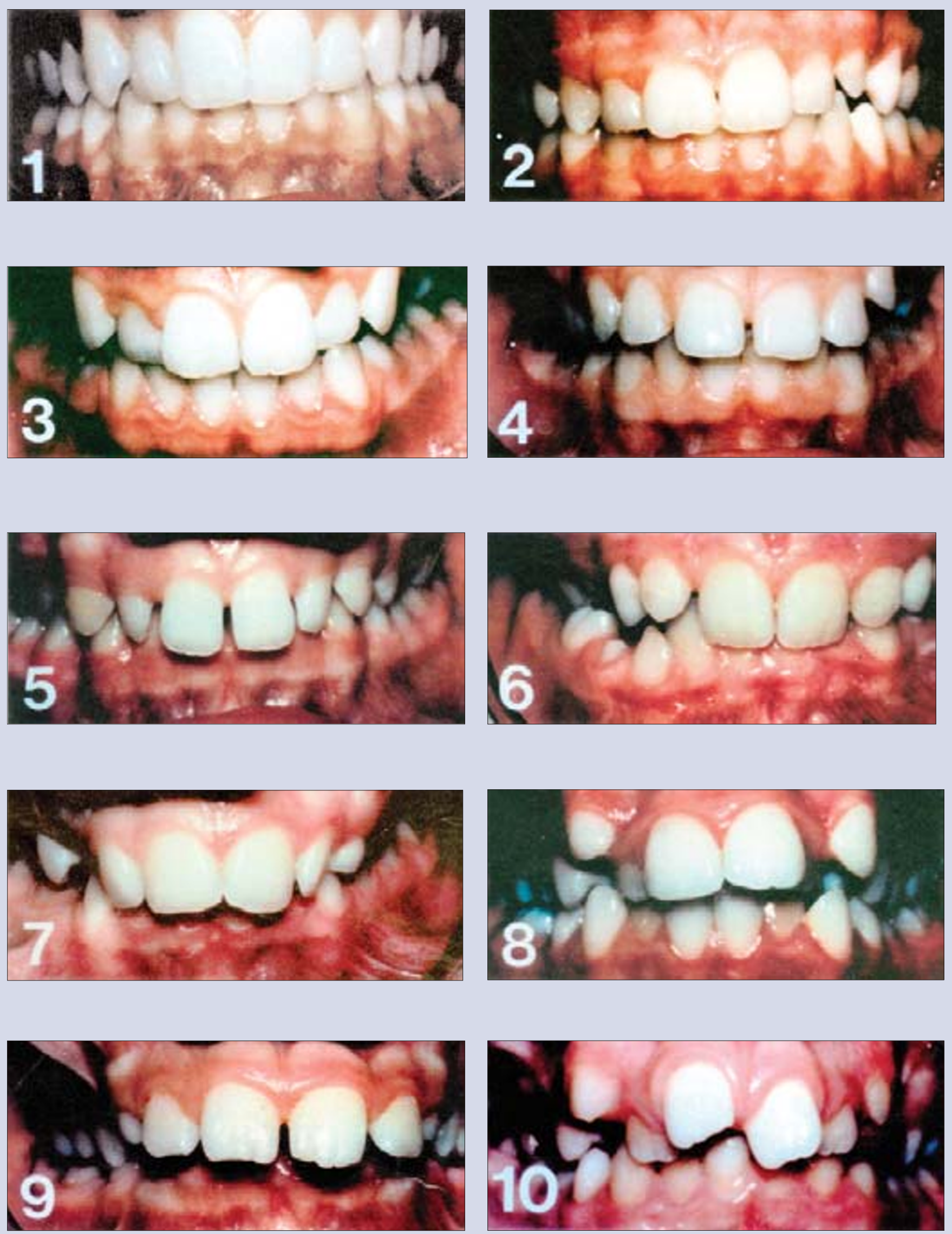